\title{
References
}

Empey, D.W, Laitinen, L.A., Jacobs, L., Gold, W.M., Nadel, J.A., 1976, Am. Rev. Respir. Dis. $113,523$.

Fryer, A.D., Maclagan, J., 1984, Br. J. Pharmacol. 83, 973.

Gies, J.-P., Landry, Y., 1988, Biochem. Biophys. Res. Comm. 150, 673.

P.we.008

\section{Adenyl cyclase activity in human alveolar macrophages}

\author{
Beusenberg *, F.D., van Amsterdam *, j.G.C., van Schaik *, J.M.E., Hoogsteden **, H.C. \\ and Bonta * I.L. \\ * Department of Pharmacology and ** Pulmonary Medicine, Erasmus University Rotterdam and University Hospital Dijkzigt, PO Box \\ 1738, 3000 DR, Rotterdam, The Netherlands
}

During the last few years substantial evidence has been raised for the involvement of inflammatory processes in the pathophysiology of Chronic Obstructive Pulmonary Diseases (COPD) and asthma. Concerning pulmonary diseases we are particularly interested in the modulatory function of the alveolar macrophage (AM). This cell plays a predominant role by exhibiting its typical inflammation-related properties as phagocytosis, cytotoxicity, antigen-presentation and the production of pro- and antiinflammatory mediators, proteolytic enzymes and oxygen-radicals. Many of these functions are known to be inhibited by increasing intracellular levels of cyclic AMP (c-AMP), a second messenger formed through the activation of adenyl cyclase. In our research, we examine the modulation of the adenyl cyclase system in alveolar macrophages with a special interest to the role of this cell in COPD and asthma. In a previous study in our laboratory, using an animal model for human allergic asthma, we observed an increase in the sensitivity of the adenyl cyclase system in antigen challenged AMs, probably the result of changes which have occured in this sysiem on the level of the regulatory binding proteins (1). In the presented study we examined the effects of some inflammatory mediators and $\beta$-adrenergic drugs on the adenyl cyclase activity of normal human alveolar macrophages. AMs were obtained by broncho alveolar lavage (BAL) of 29 healthy smoking $(n=25)$ and non-smoking $(n=4)$, male $(n=4)$ and female $(n=25)$ subjects; mean age was $34 \pm 9.4$ years. All subjects did not receive any medication during three weeks prior to BAL and had no history of pulmonary disorders. We did not exclude smoking subjects from this study as similar results were found between smokers and non-smokers in basal c-AMP levels (smokers: $2.47 \pm 0.08$ vs. non-smokers: $2.94 \pm 0.45 \mathrm{pmol} \mathrm{c}-\mathrm{AmP} / 1 \cdot 10^{6} \mathrm{cells}$ ) and c-AMP response due to agonist-exposure; the viability of the cells recovered from BAL was $90.9 \pm 0.88 \%$ whereas the majority $(>95 \%)$ consisted of AMs as differentiated by May-Grünwald-Giemsa staining. The inflammatory mediators prostaglandin $\mathrm{E}_{2}\left(\mathrm{PGE}_{2}\right)$, prostacyclin (DC-PGI 2 , a stable analogue) and histamine induced a dose-dependent increase of basal c-AMP levels in AMs (PGE 2 : 379-61\%, DC.PGI $2: 231 \pm 51 \%$, histamine: $115 \pm 35 \%$; dose-range from $2.8 \cdot 10^{-8}-2.8 \cdot 10^{-4} \mathrm{M}$ ) whereas platelet activating factor (PAF) had no effect on AM adenyl cyclase (dose-range from $1 \cdot 10^{-12}-1 \cdot 10^{-5} \mathrm{M}$ ). Using the selective histaminergic receptor antagonists Mepyramine and Cimetidine $\left(1 \cdot 10^{-5} \mathrm{M}\right)$, it was shown that the effect of histamine was mediated via $\mathrm{H}_{2}$-receptors. The non-selective and full $\beta$-adrenergic agonist isoprenaline induced an increase of basal c-AMP levels of $175 \pm 36 \%$ whereas the $\beta_{2}$-selective adrenergic agonist salbutamol induced an increase of only $76 \pm 10 \%$. Again, using $\beta$-adrenoceptor antagonists, it was shown that the effects of isoprenaline and salbutamol on AM adenyl cyclase were mediated through $\beta_{2}$-receptors. These results show that the inflammatory mediators $\operatorname{PGE}_{2}$, DC-PGI ${ }_{2}$ and histamine and $\beta$-adrenergic drugs can stimulate adenyl cyclase of human AMs whereas PAF has no effect. Furthermore it was shown that smoking seems to have no effects on the AM-adenyl cyclase activity and that human AMs possess functional $\mathrm{H}_{2}$-histaminergic and $\beta_{2}$-adrenergic receptors coupled to adenyl cyclase. Apparently, mediators and frequently used anti-asthmatic drugs (like salbutamol and anti-histaminics) have besides their action on bronchial smooth muscles an important decreasing effect on macrophage-activity. Future studies include the effects of inflammatory mediators and drugs used in the treatment of bronchial hyperreactivity on the modulation of the adenyl cyclase system in AMs of patients with COPD and asthma with a special reference to the relationship between the adenyl cyclase system, the guanyl cyclase system and arachidonic acid metabolism.

This study is financially supported by the Netherlands Asthma Foundation (NAF). 


\title{
Reference
}

Beusenberg, F.D., M.J.P. Adolfs, J.M.E. van Schaik, J.G.C. van Amsterdam and I.L. Bonta: Antigen challenge modifies the cyclic AMP response of inflammatory mediators and $\beta$-adrenergic drugs in alveolar macrophages, Eur. J. Pharmacol., in press.

P.we.009

\section{The mediators involved in airway hyperresponsiveness and airway inflammation in rat allergic asthma}

\author{
Chiba, Y., Sugiyama, Y. and Misawa, M. \\ Department of Applied Pharmacology, School of Pharmacy, Hoshi University, 2-4-41 Ebara, Shinagawa-ku, Tokyo 142, Japan
}

Bronchial hyperresponsiveness is a very important determinant for bronchial asthma. Recently, the relationship between airway hyperresponsiveness and inflammation has been attracted attention. In the present study, the changes in airway responsiveness of acetylcholine (ACh)-induced bronchoconstriction and in airway vascular permeability, after DNP-Ascaris (DNP-Asc) antigen challenge, were investigated by using a rat allergic asthma. The relationship among airway hyperreactivity, immediate asthmatic response (IAR) and airway inflammation was thus referred. Male Wistar rats were actively sensitized with DNP-Asc together with killed Bordetella pertussis vaccine as an adjuvant, according to the method of Tada and Okumura (1971). Five days later, the animals were boosted with DNP-Asc. At the eighth day after the first immunization, they were challenged by inhalation of the antigen. The experiment investigating airway hyperresponsiveness was performed as the following. At first, an asthmatic reaction was induced by aerosolized DNP-Asc (6 mgP/ml) challenge for $10 \mathrm{~min}$ in a chamber under non-anesthesia. After $24 \mathrm{hr}$, rats were anesthetized with pentobarbital $(50 \mathrm{mg} / \mathrm{kg}$, i.p.) and immobilized with pancuronium $(1 \mathrm{mg} / \mathrm{kg}$, i.v.) under artificial respiration. ACh solutions (0.003\%-0.3\%) were inhaled, in the increasing order, at intervals of 2 min with an ultrasonic nebulizer. Airway constriction was measured with a modified Konzett-Rössler method and expressed as a change in ventilation overflow (VO). In the second experiment on IAR, the response was induced by DNP-Asc challenge under anesthesia, and the airway constriction immediately evoked was measured as a change in VO. The third experiment on investigating airway vascular permeability was performed as the following. Rats were challenged with DNP-Asc under non-anesthesia. Following 1, 6 or $24 \mathrm{hr}$, they were anesthetized and i.v. administered Evans blue (E.B.; $30 \mathrm{mg} / \mathrm{kg}$ ) and heparin $(600 \mathrm{U} / \mathrm{kg})$. After bleeding and thoracotomy, the amount of E.B. extracted from airway tissues was measured by the method of Evans et al. (1987). To investigate the chemical mediators involved in the airway hyperresponsiveness, IAR and airway vascular permeability, the following drugs were administered before DNP-Asc challenge: chlorpheniramine $\left(\mathrm{H}_{1}\right.$ blocker), indomethacin (cyclooxygenase inhibitor), ONO-1078 (5-lipoxygenase inhibitor), CV3988 (PAF antagonist), OKY-046 (thromboxane $A_{2}$ synthetase inhibitor), and a combination of CV-3988 and OKY-046. At $24 \mathrm{hr}$ after DNP-Asc challenge, the airway responsiveness to $A C h$ was significantly increased. The increase was inhibited by pretreatments with ONO-1078, CV-3988 and OKY-046. Prominent IAR induced after DNP-Asc challenge in all the animals was inhibited by pretreatments with chlorpheniramine, indomethacin, CV-3988 and ONO-1078. Furthermore, airway vascular permeability was significantly increased at $24 \mathrm{hr}$ after DNP-Asc challenge. The increase was inhibited by pretreatments with ONO-1078, CV-3988 and OKY-046. From the above results, it is suggested that the chemical mediators involved in might be as follows: leukotrienes, PAF and thromboxane $A_{2}$ in the airway hyperresponsiveness; histamine, prostaglandins, PAF and leukotrienes in IAR; and leukotrienes, PAF and thromboxane $A_{2}$ in airway inflammation. A close relationship between the airway hyperresponsiveness and airway inflammation is suggested.

\section{References}

Tada, T. and Okumura, K., 1971, J. Immunol. 106, 1002.

Evans, T.W., Chung, K.F., Rogers, D.F., and Barnes, P.J., 1987, J. Appl. Physiol. 63, 479. 